\title{
A NOTE ON THE PROCESSOR-SHARING QUEUE IN A QUASI-REVERSIBLE NETWORK
}

\author{
PANTELIS TSOUCAS* AND \\ JEAN WALRAND, ${ }^{*}$ University of California, Berkeley
}

\begin{abstract}
Consider a processor-sharing queue placed in a quasi-reversible network in equilibrium. This note explains why the expected sojourn time of a customer in such a queue is proportional to his service time.
\end{abstract}

\section{The problem}

The result recalled in the abstract has been observed in the literature (see [2]).

This note shows that result to be a direct consequence of quasi-reversibility.

The situation is depicted in Figure 1. The network $N$ is quasi-reversible and node 1 is processor-sharing. This network can be open, closed, or mixed. (See [1] for definitions.)

Denote by $T$ the duration of one specific sojourn of a given customer in node 1 and by $S$ the corresponding service time in the node.

It will be shown that $E[T \mid S]=\alpha S$, where $\alpha$ is a constant to be determined from the network parameters. Notice that one then has $E\{T\}=\alpha E\{S\}$, so that it is clear how to calculate $\alpha$.

Assume that $x$ and $y$ are two positive numbers such that $\operatorname{Pr}\{S=x\}$ and $\operatorname{Pr}\{S=y\}$ are positive. If only the density of $S$ is non-zero at $x$ and $y$, then an arbitrarily small perturbation of the distribution of $S$ would lead us to the previous situation. Also, one can assume that $x / y=m / n$ for some $m$ and $n$ in $\{1,2,3, \cdots\}$.

All that has to be shown is that

$$
\frac{E[T \mid S=x]}{E[T \mid S=y]}=\frac{x}{y}
$$

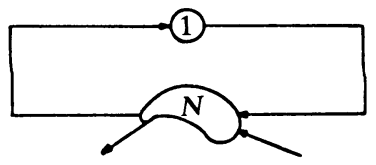

Figure 1

Received 11 February 1983.

* Postal address for both authors: Department of Electrical Engineering and Computer Sciences and the Electronics Research Laboratory, University of California, Berkeley, CA 94720 , U.S.A. 


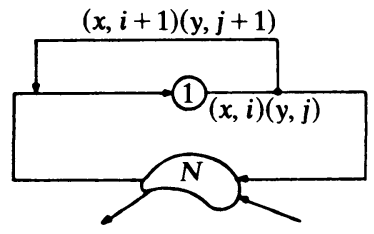

Figure 2

\section{The argument}

Let $x=m \varepsilon$ and $y=n \varepsilon$. A customer requiring $m \varepsilon$ seconds of service in node 1 can be thought of as being fed back $m-1$ times in that node and requiring $\varepsilon$ seconds during each visit.

This leads to the equivalent model of Figure 2. In that network, the customers requiring a service time $x$ are fed back $m-1$ times. This is accomplished as follows. Say that a customer is of class $(x, i)$ if his total service time is $x$ and he has been fed back $i$ times $(i=0,1, \cdots, m-1)$. With probability 1 , a class $(x, i)$ customer is fed back and becomes a class $(x, i+1)$ customer, for $i=0,1, \cdots, m-2$. A customer of class $(x, i)$ requires a service time of $\varepsilon$ in node 1 .

Similarly one defines the classes $(y, j)$ for $j=0,1, \cdots, n-1$, and their feedbacks.

The other customers proceed as before, and the customers of class $(x, m-1)$ or $(y, n-1)$ are not fed back.

The crucial observation is that upon being fed back, customers of class $(x, i)$ or $(y, j)$ find the queue in the same distribution for all $i$ and $j$. This distribution is indeed the equilibrium distribution, since the network is quasi-reversible.

Hence

$$
m^{-1} E[T \mid S=m \varepsilon]=n^{-1} E[T \mid S=n \varepsilon]
$$

and this establishes (1).

\section{Acknowledgments}

The authors wish to thank Dr D. Mitra for bringing this problem and reference [2] to their attention.

This research was supported in part by the NSF under Grant ECS-8205428.

\section{References}

[1] Kelly, F. P. (1979) Reversibility and Stochastic Networks. Wiley, London.

[2] MrTRA, D. (1981) Waiting time distributions from closed queueing network models of shared-processor systems. In Performance 81, ed. S. J. Kylstra. North-Holland, Amsterdam. 